\title{
Next Generation Simulation Tools: The Systems Biology Workbench and BioSPICE Integration
}

\author{
HERBERT M. SAURO, ${ }^{1,2}$ MICHAEL HUCKA, ${ }^{2}$ ANDREW FINNEY, ${ }^{2}$ \\ CAMERON WELLOCK, ${ }^{1}$ HAMID BOLOURI, ${ }^{2,3}$ JOHN DOYLE, ${ }^{2}$ \\ and HIROAKI KITANO ${ }^{4}$
}

\begin{abstract}
Researchers in quantitative systems biology make use of a large number of different software packages for modelling, analysis, visualization, and general data manipulation. In this paper, we describe the Systems Biology Workbench (SBW), a software framework that allows heterogeneous application components-written in diverse programming languages and running on different platforms-to communicate and use each others' capabilities via a fast binary encoded-message system. Our goal was to create a simple, high performance, opensource software infrastructure which is easy to implement and understand. SBW enables applications (potentially running on separate, distributed computers) to communicate via a simple network protocol. The interfaces to the system are encapsulated in client-side libraries that we provide for different programming languages. We describe in this paper the SBW architecture, a selection of current modules, including Jarnac, JDesigner, and SBWMetatool, and the close integration of SBW into BioSPICE, which enables both frameworks to share tools and compliment and strengthen each others capabilities.
\end{abstract}

\section{INTRODUCTION}

$\mathbf{T}$ The aPPlication of MATHEMatics and computer science to understanding biochemical networks has a long history, going back in fact to the initial development of computers in the 1930s and 1940s (Chance et al., 1962; Burns, 1971). More recently and especially since the development of high-throughput data collection and the completion of the human genome project, there has been a renewed and vigorous interest in understanding the dynamic aspects of cellular networks (Endy and Brent, 2001; Rao and Arkin, 2002; Tyson et al., 2003). Although it has been appreciated for many years that cellular networks were dynamic, intricate control systems, the molecular biology revolution of the last 30 years, with its focus on DNA and protein structure, has taken center stage in mainstream biology at the expense of other studies. It is only in the last few years that "quantitative systems biology" is finally beconing a mainstrean topic in biology.

One of the important techniques at the disposal of the quantitative systems biologist is computer model-

\footnotetext{
${ }^{1} 1$ Keck Graduate Institute, Claremont, California.

${ }^{2}$ Control and Dynamical Systems, Calfornta Instizute of Technology, California.

${ }^{3}$ Institute of Systemas Biology, Seattle, Washington.

ERATO Kitano Symbiotic Systems Project, Ingumae Shibuya-Ko, Tokyo, Japan.
} 


\section{SAURO ET AL.}

ling. This involves constructing kinetic models of the biochemical reaction networks, incorporating network as well as kinetic information. The models can vary in size from very small models comprising only two reaction steps to whole cell models incorporating hundreds of reaction steps. The models are studied by computing the time-course behavior or the steady state. By these means, hypotheses can be tested, new hypotheses developed and a general understanding of the network's behavior developed.

Almost from the earliest days of simulation, it was realized that developing the necessary mathematical models was tedious and error prone. As a result, specialized software was developed to help users input the models into the computer. This involved allowing users to enter reaction networks in a familiar form, often as a list of reactions and kinetic laws. This approach has been followed ever since. Interestingly, though perhaps not surprisingly, the software tools themselves have tended to progress in step with technological developments. In the early years of modelling, tools took a script-based approach to specifying models (Garfinkel, 1968; Park and Wright, 1973; Fell and Sauro, 1990; Sauro and Fell, 1991). With the beginning of the widespread use of graphical user interfaces in the 1980s, simulation tools took a marked change in direction. Instead of specifying models using text-based script files, users could now specify models using much friendlier GUI-based user interfaces. The most famous of this new generation was and still is, Gepasi, developed by Pedro Mendes (Mendes, 1993). The development of Gepasi began a new episode in software development, which continues to the present day, and there are now numerous tools available that take a similar approach.

Easier you use, GUI-based simulators tend to be less flexible compared to script-based tools. In fact many general purpose commercial simulation tools are script based for this very reason (e.g., Mathematica, Matlab, MathCAD). As a result, script-based tools have continued to be developed, the most advanced example of this being Jarnac, which incorporates a full programming language as well as extensive libraries for numerical analysis. In more recent years, a second generation of GUI-based tools has also emerged that take the user interface to an even more visual level. That is, models in the form of networks are drawn on a canvas and the diagrams converted into a mathematical representation for simulation. Examples of such tools include JDesigner CellDesigner, and KinCyte. At the last count, there were over 33 different packages for simulating cellular networks. This proliferation of tools has resulted in a variety of capabilities and interfaces. However, though welcome in many respects, this proliferation has resulted in two unwelcome side effects:

1. Each tool uses its own format, often undocumented, to store models. The result is that a model saved in one tool cannot be loaded into another. This obviously hinders the free exchange of models from one tool to another.

2. The second problem is that many of the tools duplicate each other's capabilities. Writing simulation tools takes time, and many of the projects are short-lived, which means that the authors are unable to develop the tools much further than basic functionality. As a result, many of the tools provide similar functionality.

Untike other software development communities, there is little tradition of code reuse in the system biology community. As a result, the community has seen much duplicated effort and little true novelty.

The first problem, that of model exchange, has been addressed by introducing a standard format for all tool writers to employ. This standard is called Systems Biology Markup Language (SBML) (Hucka et al., 2003). Along with CellML (Hedley et al., 2001), the introduction of a standard format is beginning to make a significant impact on tools writers, and the majority of the most widely used tools now employ SBML as a means to exchange models.

The second issue is more difficult to address, that is how to encourage code reuse in the community. Our attempt to resolve this has been to develop a software framework called the System Biology Workbench. The workbench allows different tools to expose programmatic functionality to other tools. This means that a developer can now build on previous work without having to understand in detail the often intricate internal workings of other tools. All a developer need know is the interface that the tool exposes. Thus, a particular tool may expose a time-dependent simulation interface from a simulation tool, another tool developer-rather than invent another simulation tool-can explot this capability and develop a new tool that can carry out additionnl hunctions. The workload for the second developer is greatly reduced, and they can instead concentrate on novel functionality. 


\section{NEXT GENERATION SIMULATION TOOLS}

BioSPICE takes a very similar approach, so much so that both SBW and BioSPICE are becoming closely integrated. Like SBW, the goal of the BioSPICE project (BioSPICE, 2001) is to create an open source framework and toolset for modelling dynamic cellular network functions. The hope is that this will develop a user community committed to using and extending the tools. Clearly, the SBW project has considerable overlap with the BioSPICE project. We are currently developing a software bridge that will allow modules in both SBW and BioSPICE to communicate with each other. At the moment, SBW and BioSPICE are to a large extent complimentary in functionality; whereas BioSPICE is more data centric, SBW's emphasis is on analysis. As a result, SBW can provide a range of ready-made modules to the BioSPICE program, including simulators, both stochastic and deterministic, model buiding tools, network analysis tools (based on METATOOL [Pfeiffer et al., 1999]), and as part of the BioSPICE program, tools for optimization and bifurcation analysis. Such a bridge would therefore clearly benefit both communities.

A number of documents have been published in the past on SBW (Hucka et al., 2002), but none have focused on the internal workings of SBW or on some of the applications that we have developed in conjunction with SBW. In this paper, we will focus on these issues. In particular, we will describe the data structures and the mode of operation of SBW, tools such as Jarnac, JDesigner, and Metatool, and how SBW will be integrated into BioSPICE.

\section{MATERIALS AND METHODS}

The Systems Biology Workbench is a computational resource sharing framework. It allows applications to communicate with each other efficiently and without loosing their identity. Applications can be written in a variety of different languages and can run on different operating systems across the internet. The entire workbench is open-source and vendor independent. SBW was designed to offer excellent performance and be geared specifically towards scientific applications.

\section{SBW architecture}

In setting out the requirements for SBW, the following items were our highest priority:

- Simplicity: The framework must be simple enough that interested developers can use it in their projects with a minimum amount of learning and coding effort. We considered here the full range of developers, from experienced to novice.

- Performance: Since SBW will be used for scientific work, performance was an important issue. Moving data from module to module has to be efficient.

- Component modularity: As new tools and methods are developed, it must be possible to implement them as modules that can be hooked into the existing framework without having to modify the framework itself.

- Language interoperability: The framework must support the interaction of modules written in different programming languages.

- Free distribution: All interested users must be able to obtain both SBW and its source code for free. Any software that is incorporated into SBW and distributed with it, such as GUI widgets or object libraries, must itself be free of licensing fees or restrictions on redistribution. (This is only a requirement on SBW itself, and not on modules built for SBW or other software developed using SBW.)

- Portability: The framework must be portable to Microsoft Windows (NT, 2000, XP) and Linux initially, and clearly be portable to other platforms in the future.

- On-demand plug-in loading: Modules that implement particular capabilities should not have to be preloaded into SBW every time it is started; instead, the system should be data- and task-driven and dynamically load modules on an as-needed basis. This helps keep the size of the ruming system to a minimuin.

- Support for distributed conputing: The user should have control over where processes are executed and the ability to interact with remote services. 


\section{SAURO ET AL.}

Given the requirements above, the question then arose, what software technology should we employ to build the framework. Some of the requirements immediately eliminated certain existing frameworks, including DCOM because it is limited to Microsoft Windows platforms, and Java JNI and Java RMI because this would limit the framework to Java.

Other frameworks such as XML-RPC (Winer, 2001) or SOAP (Box et al., 2000) were also unsuitable, because these frameworks did not meet our performance criteria. Some recent studies in particular (Olson and Ogbuji, 2002) indicate that SOAP and XML-RPC are orders of magnitude slower compared to CORBA or simple socket transmission.

CORBA was another possibility (OMG, 2001). However, CORBA is notorious for being difficult to master and requires highly skilled programmers to work with. Hence, CORBA was not in line with our first requirement, that of simplicity. Since the development of SBW, Microsoft has released .NET, which in some limited respects is similar to SBW. The .NET framework has many of the desirable features we sought in the requirements; however, it has an uncertain future due to its availability on only a single platform, although there is now an open-source, platform independent variant called Mono.

Since we couldn't find a suitable existing framework that satisfied all our requirements, it was decided to develop our own. During the period when we were considering the design, peer-2-peer technologies were becoming a fashionable and useful mode of communication (Oram, 2001). Peer-2-peer possessed many of the attributes that were attractive to us. The three main features that stood out were simplicity, performance, and language independence. Most peer-2-peer frameworks were characterized by binary transmission of data over simple TCP/IP sockets. In addition, they were also characterized by simple APIs, which helped ensure their rapid take up by third-party developers as witnessed by the plethora of peer-2-peer clients. As a result of these observations, it was decided to base SBW on a binary messaging passing architecture over TCP/P sockets.

Architecture. SBW uses a broker-based, message-passing architecture that allows dynamic extensibility and configurability. Software modules in SBW interact with each other as peers in the overall framework. Modules are started on demand through user requests or program commands. Modules are executables which have their own event loops and all remote calls run in their own threads. As shown in Figure 1, interactions are mediated through the SBW Broker, a small program running on a user's computer. The Broker enables locating and starting other modules and establishing communications links between them. Communication is implemented using simple TCP/IP sockets, which are fast and lightweight, with a straightforward programming interface.

Broker-based architectures are a means of structuring a distributed software system with decoupled components that interact by remote service invocations. In SBW, the remote service invocations are implemented using message passing. Because interactions in a message-passing framework are defined at the level of messages and protocols for their exchange, it is easier to make the framework neutral with respect to imptementation languages and platforms: modules can be written in any language, as long as they can

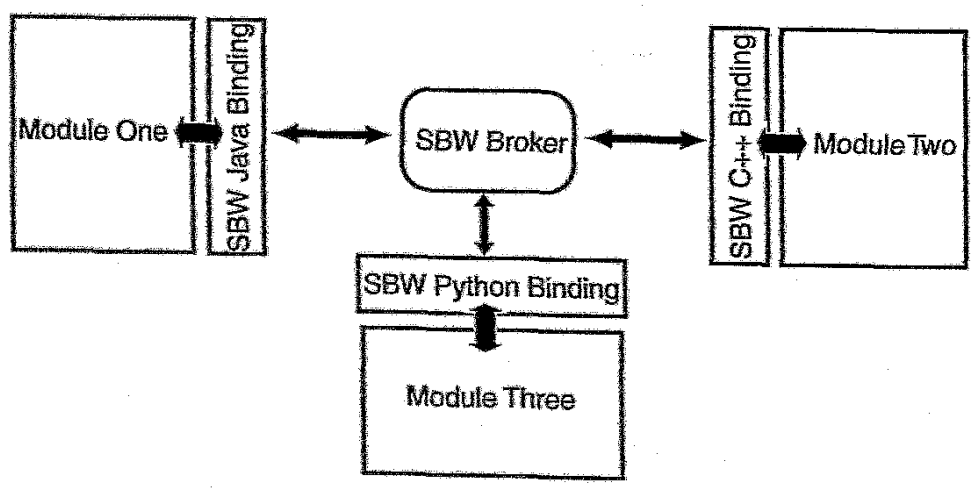

TIC, 1 . Comection between broker, modules, and binding libraries. Packing and unpacking of messages is handled
by the banding libraries. 


\section{NEXT GENERATION SIMULATION TOOLS}

send, receive, and process appropriately structured messages using agreed-upon conventions. The organization of SBW means that modules can be easily exchanged, added, or removed, even at run-time, under user or program control.

We strove to develop an API for SBW that provides a natural and easy-to-use interface in each of the different languages for which we have implemented libraries. By "natural," we mean that it uses a style and features that programmers accustomed to that language would find familiar. For example, in Java, the high-level API is oriented around providing SBW clients with proxy objects whose methods implement the operations that another application exposes through SBW.

An SBW module provides one or more interfaces or services. Each service provides one or more methods. Modules register the services they provide with the SBW Broker. The module optionally places each service it provides into a category. By convention, a category is a group of services from one or more modules that have a common set of methods.

Supported languages and operating systems. One of the key advantages of SBW is its language and OS neutrality. At this point in time, we have support for Windows and Linux operating systems (MacOS is scheduled for future development). The languages we support, through language bindings, include Java, $C$, $\mathrm{C}++$, Delphi, C\#, VB.NET, Python, and Perl. There are developments currently underway to create bindings for Matlab and Mathematica.

Capabilities. Here we summarize the capabilities of SBW:

- Dynamic service and module discovery: The SBW Broker keeps track of modules, services, and service categories, and provides facilities for a module to learn about them.

- Remote method invocation: The bread and butter of SBW is enabling one module to invoke a service method in another module. If necessary, the SBW Broker will automatically start an instance of a module whose services are requested.

- Data serialization: Method invocations involve sending messages between modules, with arguments and data packed into message streams. For some languages such as Java, Delphi, C\#, VB.NET, Perl, and Python, the SBW library provides proxy objects that hide the message-passing, so that, to client programs, remote services appear as local objects whose methods can be invoked like any other object method in that language.

- Exception handling: SBW provides facilities for dealing transparently with exceptional conditions.

- Event notification: Certain events in SBW, such as the startup or shutdown of an instance of a module, are announced to all modules upon their occurrence.

- Module, service and method registration: Modules that are not running but wish, nevertheless, to advertise their services can do so by registering with the broker. This is accomplished by runuing the module once, in a special mode. The registration facilities allow a module to record with the Broker the services that the module provides, the command that should be used to start up the module on demand, and other information. The SBW Broker stores this in a disk file, so that the information provided by modules is persistent between start-up and shutdown of the modules and the Broker.

Messaging protocols. At the heart of SBW is the messaging protocol used to exchange information between the different modules. For efficiency reasons, messages that are exchanged between modules are simple sequences of binary data. For each programming language, there is a language binding library that takes care of much, if not all, of the housekeeping necessary to operate through SBW, including connection and transmission of data. In addition, issues such as little and big-endian byte ordering need not concern the developer as this is taken care of automatically by the binding libraries. Each binding also provides the necessary message packing and unpacking logic and exposes functionality in the form of an easy-to-use API (Fig. 1).

All modules that make a connection to the SBW Broker are assigned a numeric identiffication handle. The handle is generated when a modnle makes its initial connection with the SBW broker or when SBW starts a module and makes a connection. The Broker itself bas its own publicly reserved handle that allows 
SAURO ET AL.

\begin{tabular}{|c|c|c|c|c|c|c|c|}
\hline & \multicolumn{6}{|c|}{ Header: 25 bytes } & Variable Length \\
\hline $\begin{array}{l}\text { Length } \\
\text { (32-bit) }\end{array}$ & $\begin{array}{l}\text { Destld } \\
\text { (32-bit) }\end{array}$ & $\begin{array}{l}\text { Type } \\
\text { (byce) }\end{array}$ & $\begin{array}{l}\text { UID } \\
\text { (32-bit) }\end{array}$ & $\begin{array}{c}\text { Srcld } \\
\text { (32-bit) }\end{array}$ & $\begin{array}{l}\text { Serviceld } \\
\text { (32-bit) }\end{array}$ & $\begin{array}{l}\text { Methodld } \\
\text { (32-bit) }\end{array}$ & Data Payload \\
\hline
\end{tabular}

FIG. 2. Structure of the send/call message.

modules to make requests to services provided by the Broker. When a module wishes to communicate to another module, it does so by sending a message through the Broker. The message will contain the destination module handle that the Broker will use to route the message onto the appropriate module.

There are four basic message types: messages that represent blocking calls to methods in other modules or to the broker itself, messages that represent non-blocking calls to methods in other modules or the broker itself, messages that represent replies to earlier messages, and messages that represent error conditions as a result of poorly formatted messages or exceptions that occur in modules.

Call and send messages. These messages come in two varieties, send (non-blocking) and call (blocking). Both types of message have the same internal structure. What distinguishes the two is the value of the message type byte (Fig. 2).

The fields in a call/send message have the following meanings:

Length: Length of the message in bytes, including the length integer itself.

Destld: A handle which indicates the destination module for this message.

Type: Indicates whether the message is a call, send, reply or an error condition.

UID: A unique identifier associated with this message. A corresponding reply will have the same UID (Unique identifier) and can be used to match a reply to the original sender.

Srcld: A handle which indicates the source module for this message.

Serviceld: Indicates the required service.

Methodld: Indicates the particular method in the service.

Data payload: A data payload containing the arguments required by the method.

Reply messages. A reply message is sent in response to a call message. Its sole purpose is to deliver raw data to the recipient as a result of a method call. The format of the first 13 bytes of a reply message is identical to a calling message except that the type byte is set to the reply message type. All remaining data in the reply message is composed of data returned by the call (Fig. 3).

Error messages. Etror messages are sent in response to an error condition originating either as a result of a badly formatted message or as a result of an exception in the method which was meant to service the message. The error byte is a byte to indicate the type of error, these are defined in the developer documentation at the main SBW web site (Fig. 4).

\section{Data types}

In the previous section, we described the structure for the four different SBW messages types. The call and send messages include an optional data payload, which may be required by the recipient. Likewise, a

\begin{tabular}{|c|c|c|c|c|}
\hline \multicolumn{4}{|c|}{ Header 13 byes } & Variable Length \\
\hline $\begin{array}{l}\text { Lengith } \\
\text { 62-biit }\end{array}$ & $\begin{array}{l}\text { Destid } \\
\text { (32-bit) }\end{array}$ & $\begin{array}{l}\text { Type } \\
\text { (byse) }\end{array}$ & $\begin{array}{c}130 \\
(32-b x)\end{array}$ & Data Payload \\
\hline
\end{tabular}

IIG. 3. Structure of the reply message. 


\section{NEXT GENERATION SIMULATION TOOLS}

Header: 14 bytes
\begin{tabular}{|c|c|c|c|c|c|c|}
\hline $\begin{array}{c}\text { Length } \\
\text { (32-bit) }\end{array}$ & $\begin{array}{c}\text { Destld } \\
\text { (32-bit) }\end{array}$ & $\begin{array}{c}\text { Type } \\
\text { (byte) }\end{array}$ & $\begin{array}{c}\text { UID } \\
\text { (32-bit) }\end{array}$ & $\begin{array}{c}\text { Error } \\
\text { Byte }\end{array}$ & $\begin{array}{c}\text { Readable Error } \\
\text { Message }\end{array}$ & $\begin{array}{c}\text { Detailed Error } \\
\text { Message }\end{array}$ \\
\hline
\end{tabular}

FIG. 4. Structure of the error message.

reply message may also include a data payload for the recipient. In order for data to be easily exchangeable between modules, we needed to decide on a collection of defined data types. Obviously, it would not be possible to imagine every possible type of data type that a module might wish to package and send to another module; therefore, we devised a set of data types, of sufficient generality, from which any other data type could be constructed. In the first version of SBW, we defined seven basic data types. Five of these are fundamental data types, such as byte, Boolean, integer, double, and string. The remaining two are structured data types that provide the most flexility; these include arrays and lists (Table 1).

Byte. Bytes start with a byte code (dtByte) indicating a byte type. This is then followed by an 8-bit byte value.

Integers. Integers start with a byte code (dtInteger) indicating an integer type. This is then followed by a signed 32-bit integer value in Intel-byte order that has the range -2147483648 to 2147483647 .

Double. Double values start with a byte code (dtDouble) indicating a double type. This is then followed by a floating-point value stored in standard IEEE standard 754 double 64-bit format-that is, 1-bit sign, 11-bit base 2 exponent, and 52-bit fraction in Intel-byte order (Fig. 5).

Boolean. Boolean values start with a byte code (dtBoolean) indicating a Boolean type. This is then followed by a further byte indicating the value of the Boolean. A byte value of zero indicates False, and a value of one indicates True.

String. String values start with a byte code (dtString) indicating a string type. This is then followed by an unsigned 32-bit integer denoting the number of bytes in the string. The remainder of the data consists of the sequence of characters that make up the string. Note that the string is also null terminated (Fig. 6).

Arrays. Arrays are multi-dimensional objects of arbitrary size containing homogeneous data. Arrays start with a header made up of one byte indicating the data type stored in the array, and an integer indicating the number of dimensions, followed by a sequence of integers, one for each dimension, denoting the number of elements in each dimension. The header is therefore $(2+4+4 d)$ bytes long, where $d$ equals the number of dimensions of the array. Array access can be optimized at the module if it is known that the data

Tabee 1. Data Trees

\begin{tabular}{|c|c|c|}
\hline Data type & Type code & Description \\
\hline Byte & dtByte & Simple Byte \\
\hline Integer & dtriteger & 32 bit integer \\
\hline Double & dtDauble & IEEE 754 donble 64 bit format \\
\hline Boolean & dtBoolean & Byte indicating true or fatse (0 represents false) \\
\hline String & dtString & $\begin{array}{l}\text { Sequence of characters, the hirst unsigned integer } \\
\text { nuticates the length of the string }\end{array}$ \\
\hline Array & dtArray & Homogeneous array of data ( $\mathrm{n}$ dimensional) \\
\hline List & dtList & Heterogenous, pested list structure \\
\hline
\end{tabular}




\section{SAURO ET AL.}
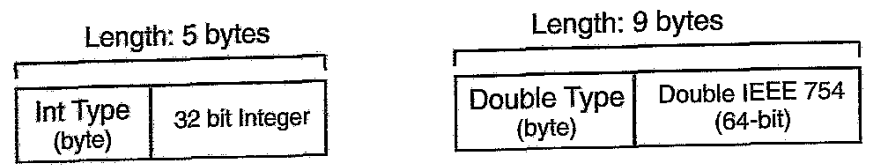

FIG. 5. Integer and double data types.

type has a fixed size. This is especially the case for simple types such as integers and doubles. In these cases, the application can carry out block copies of the data in order to greatly improve performance (Fig. 7).

Lists. Lists are recursively defined structures for storing heterogeneous data. This means that lists can be used to store other lists which allows complex relationships to be represented.

A list is a much simpler structure that an array. A list starts with a list type byte, followed by a 32-bit integer indicating the number of items in the list. Each item in the list can be any of the data types previously described, including a list (Fig. 8).

For example, the following are legal list structures:

\section{$[1,2,3,4]$}

$[1, " A T P ", 3.1415,\{1,2,3\}]$

[ [ "S1", "S2", "S3", [4,5,6]], "k1", "k2"]

$[$ ["J1", [ ["X0"], ["S1"] ], "k1S1"], ["J2", [ ["S1", "S2" ], ["S3"] ], "k2S2" ], ... ]

Note the nested lists in the third and fourth examples.

\section{Event support}

SBW supplies a number of special method calls to modules that are sent when certain events occur. These include the following:

void onModuleShutdown(Module module): This method on the listener is called every time a module instance somewhere in the SBW system disconnects from the SBW Broker. The module passed to the method represents the module instance that has just shut down.

void onModuleStart(Module module): This method on the listener is called every time a module instance starts up or connects to the SBW Broker. The module passed to the method represents the module instance that has just started.

woid onRegistrationChange(): This method on the listener is called whenever a registration change for a module occurs in the SBW Broker. Registration changes are a module registering itself with the Broker, a module registering a service with the Broker, or a module being unregistered.

\section{Simple example}

Having described in some detail the internal structures and design of SBW, it is now worth showing a sixuple example to illustrate how it might be used. The following example shows how to set up a module

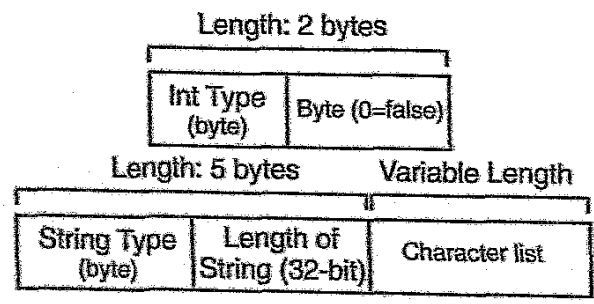

ITG. 6. Boolean and string data types. 


\section{NEXT GENERATION SIMULATION TOOLS}

Length: $2+4+4 d$ bytes

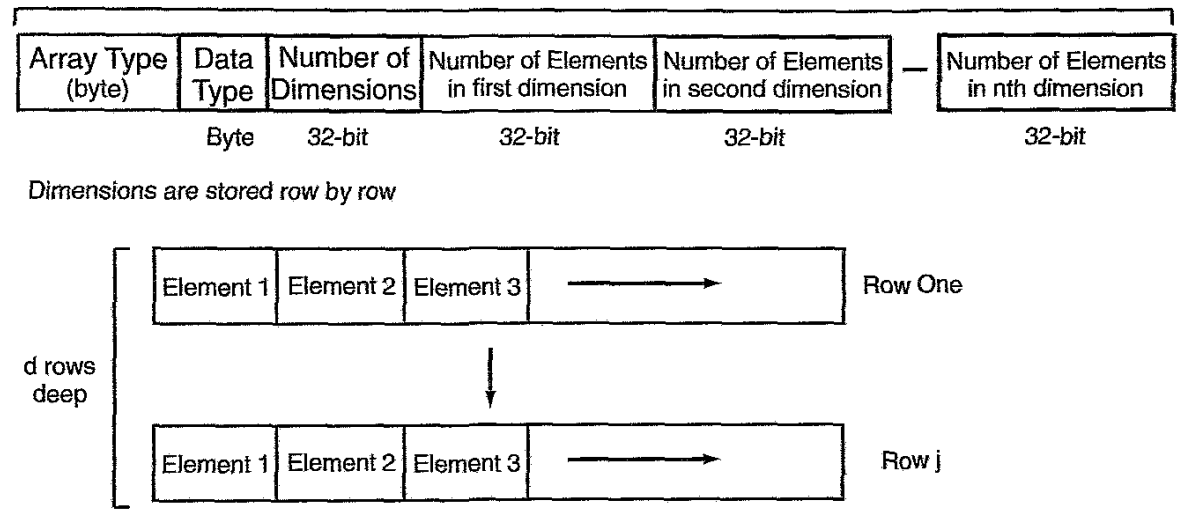

FIG. 7. Array data type.

which provides two math services, trig and $\log$, and another module which uses these services. The code is shown using Java but similar code would apply to other languages.

We first declare the classes which represent the services that the module is going to provide. In this case we will provide two services, one that offers basic trigonometric functions, and another that provides basic logarithmic functions.

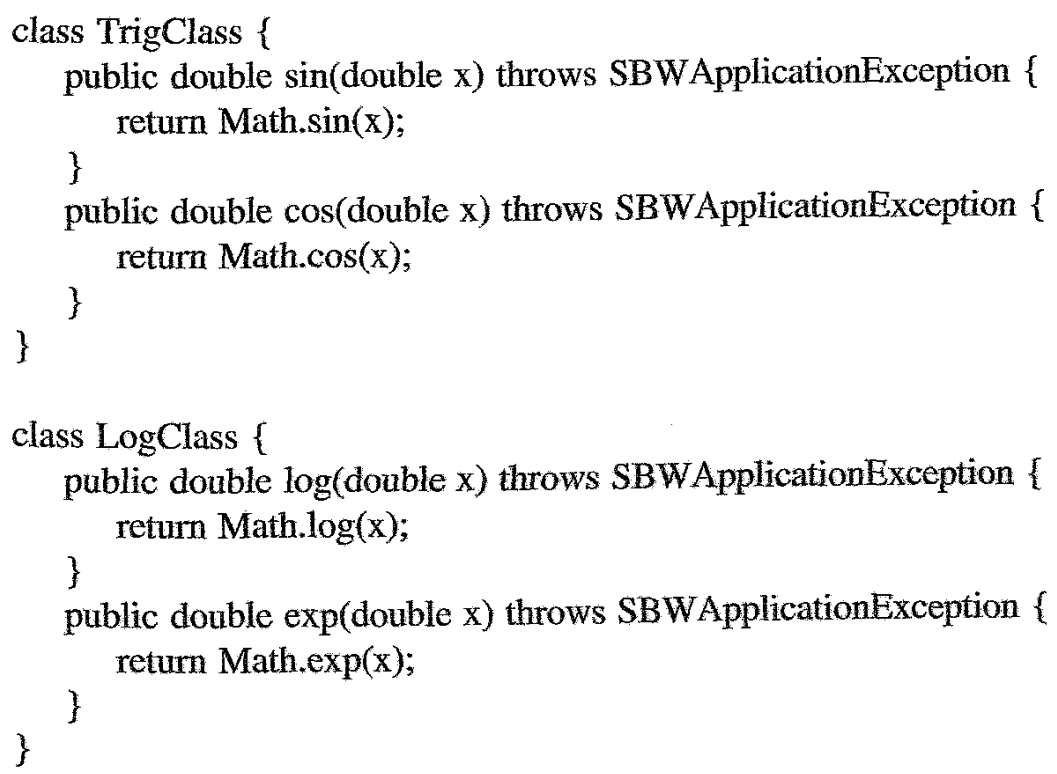

In the main program, we create a new instance of an object that represents the object that other modules will see. Into this object, we register the two services that we wish to provide. Finally, we call the run

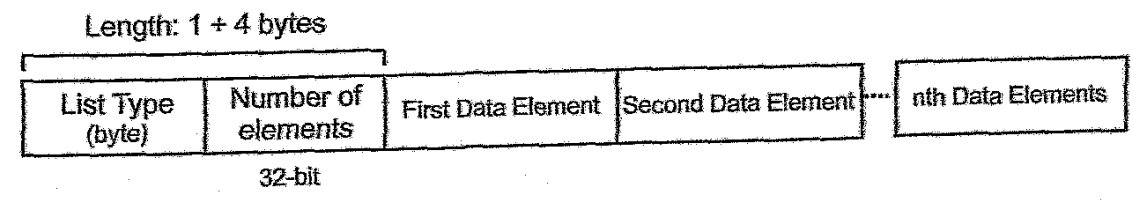

The size of each data elenent is data type dependent Data elements can be any of the standard ypes, byte, boolean, integer, double, string, arrays and hists

FIG. 8. Eist data type. 


\section{SAURO ET AL.}

method of the module object, which initiates the connection to the SBW broker. Once the broker receives the connection request, it transmits a startup event to all connected modules indicating that a new module is available. At this point, modules may now interrogate the new module and use the services that the module provides.

ModuleImpl module = new ModuleImpl("edu.caltech.math", "math", ModuleImpl.UNIQUE);

module.addService("Trigonometry", "trig functions", TrigClass.class);

module.addService("Logarithmic", "log functions", LogClass.class);

module.run(args);

The argument args in the run method is the command line argument that invoked the module. If a remote module wishes to use the services provided by math, it would use the following code:

Interface ITrigService \{

double sin(double $\mathrm{x}$ ) throws SBWException;

double cos(double $x)$ throws SBWException;

\}

Interface ILogService \{

double $\log ($ double $x)$ throws SBWException;

double exp(double $x$ ) throws SBWException;

Module module $=$ SBW.getModulefnstance("edu.caltech.math");

$/ /$ Get the individual services

Service trig = module.findServiceByName("Trigonometry");

Service log = module. findServiceByName("Logarithmic");

// Create proxy with this interface and call it:

TrtigService trigService = trig.service.getServiceObject(TrigService.class);

HogService logService = log.service.getServiceObject(ILogService.class);

Double result;

Double $\mathrm{x}=12.2$;

result $=$ trigService.sin $(x)$;

result $=\log$ Service $\log (\mathrm{x})$;

The first two sections declare interfaces of the services that will be used. In this case, the services are hardcoded, but SBW also allows runtime reflection on a remote module and thus allows methods and services to be used dynamically if need be. Under Java, the easiest approach to access remote services is to use interfaces.

Once the interfaces have been declared, a call is made to obtain a handle of the module. If the module has been registered with the Broker but is not currently running, this call will cause the module to be autontatically started up.

Finally, the objects representing the individual services are created using the getServiceObject method call. Last but not least, the methods are called through the service object returned previously.

Thus, it only takes a lew lites of code to access and call remote methods. For more details of the API, the reader is refered to the API manual available on the SBW web site. Note that services and methods on remote modutes are also avallable via a umber of interactive scripting tools, in particular Python and Perl. In these cases, interaction is even simpler, as the scripting tools will autoniatically wrap remote services into Python and Perl objects. Thus, under Python, to access the trigonometric method, sin, only requires a single line: 
print edu_caltech_math.Trigonometry.sin (30.0)

\section{The BioSPICE/SBW bridge}

One of BioSPICEs' main interfaces is based on the Netbeans IDE. This has enabled the developers of BioSPICE to implement a data flow GUI that allows users to direct data from one module to another through a GUI interface. This allows users to chain a series of processes in whatever fashion suits their needs. The BioSPICE modules themselves are made accessible to the Netbeans IDE either via OAA (Open Agent Architecture) or directly into the Netbeans IDE itself.

Work is nearing completion to construct a software bridge between SBW and OAA, which will enable clients of either system to access the services provided by the other system. These services will be made to appear as native services, identical to any other. This is made possible by a special generic translation layer.

OAA is based on a Prolog programming model and is organized in terms of agents that provide specific functions. Parameters are untyped and may be numbers, strings, lists, and several other Prolog-specific data types. Prolog functions do not have return values; rather they use a system called "unification," wherein unbound variables are replaced with results. For example, to get the sum of two numbers in Prolog, one might call a function as follows:

$\operatorname{sum}(2,5, X)$

and get the result

$\operatorname{sum}(2,5,7)$

SBW, in contrast, uses a more common model for methods, which take typed parameters and have a single typed result, that is,

int sum(int,int)

The bridge handles the conversion of the method signatures between OAA and SBW automatically. If there was a method in OAA such as get_some_data_3 (the name, 'get_some_data, 'and three parameters), this would be translated into an appropriate SBW method signature:

$$
\text { get_some_data0 }
$$

The most recent version of OAA provides for typed, directional parameters. If get_some_data_3 had two input parameters of integer type, and one output parameter of double type, then the SBW method signature would be as follows:

$$
\text { double get_some_data(int,int) }
$$

In the other direction, an SBW method such as

$$
\text { int get_just_an_int(int) }
$$

would be translated by the bridge into an OAA method get_just_an_int_2-the second parameter is just the SBW function's return value.

Incompatible data types are mapped whenever possible to equivalent types. As an example, arrays, which are used by SBW, are not recognized in OAA. These are ranslated into lists or lists-of-lists, which OAA can handle. Services in SBW appear as separate agents in OAA, and agents in OAA are represented as services of a single module (the "oaabridge") module in SBW (Fig. 9).

\section{APPLICATIONS}

In this section, we will describe an application of SBW that utilizes three SBW-compliant tools: Jamac, Designer, and METATOOL. 


\section{SAURO ET AL.}

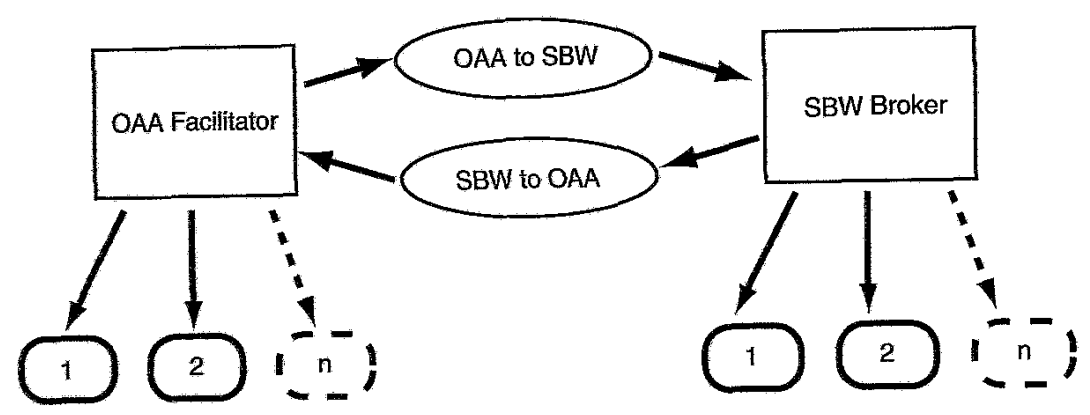

FIG. 9. Structure of the BioSPICE/SBW bridge.

\section{Jarnac}

Jarnac is a script-based simulation tool that can operate either interactively via a console window or as a simulation server for SBW. Details of Jarnac can be found in Sauro (2000). Here we will just describe the SBW interface.

The Jarnac SBW interface supports four services: modelServices, msim, mat, and asim. asim is used to interface to the SBW GUI, details of which can be found at the SBW website (www.sbw-sbml.org). The interface provided by msim is more extensive than asim and is the one described here.

msim supports multiple simulation instances; that, is more than one simulation can be active at any one time. All the methods in msim require a model handle to indicate the current model instance. Model instances can be created and destroyed through the modelServices service. msim provides a range of methods to control, interrogate, and simulate either continuous (ordinary differential equation based), or probabilistic (based on the Gillespie method) models.

Models are loaded into a model instance in the form of SBML Level 1 (Hucka et al., 2003) via the load SBMLO method. A range of access methods are provided to interrogate the currently loaded model. For example, remote modules can request the number of reactions, the rates of change of species, and the reaction velocities. In addition, methods are provided for a remote application to access the model equations, including the list of differential equations, the rate law expressions (both in infix format), and the list of any conservation laws in the model. Methods are also provided to allow a remote application to modify parameters and initial conditions. Finally, there is a range of analysis methods, including time course simulation, Gillespie stochastic simulation, steady-state analysis, sensitivity analysis, and specialist information such as the Jacobian matrix.

The remaining service, mat, supplies two matrix-related methods: one method to compute the eigenvalues for a matrix and a second method to compute the inverse for a matrix.

Jamac can be nun in two modes, either interactively, where a user has access to the model capabilities through a console window and via the SBW interface, or in server mode, where Jarnac runs invisibly as a background service. The only way to access Jarnac in server mode is via the SBW interface.

Note that we provide a GUI-based browser tool that allows a users to inspect the services and methods available from a particular module.

\section{JDesigner}

JDesigner is a model design tool for editing biochemical networks visually. It has no simnlation capabilities itself but it can interface to the Jarnac SBW interface. Unlike Jarnac or SCAMP (Sauro and Fell, 1991; Sauro, 2000), where models are entered in the form of a script describing the chemical reactions and rate laws, under JDesigner, models are entered visually as reaction networks. JDesigner stores models in the form of SBM. Level 1 (Hucka et al, 2003) with specific annotation added to support layout informawion. Detalts of this and other information on JDesigner can be found at the website (www.sys-bio.org). Figure 10 illustrates a basie sereen shot from Jesigner. 


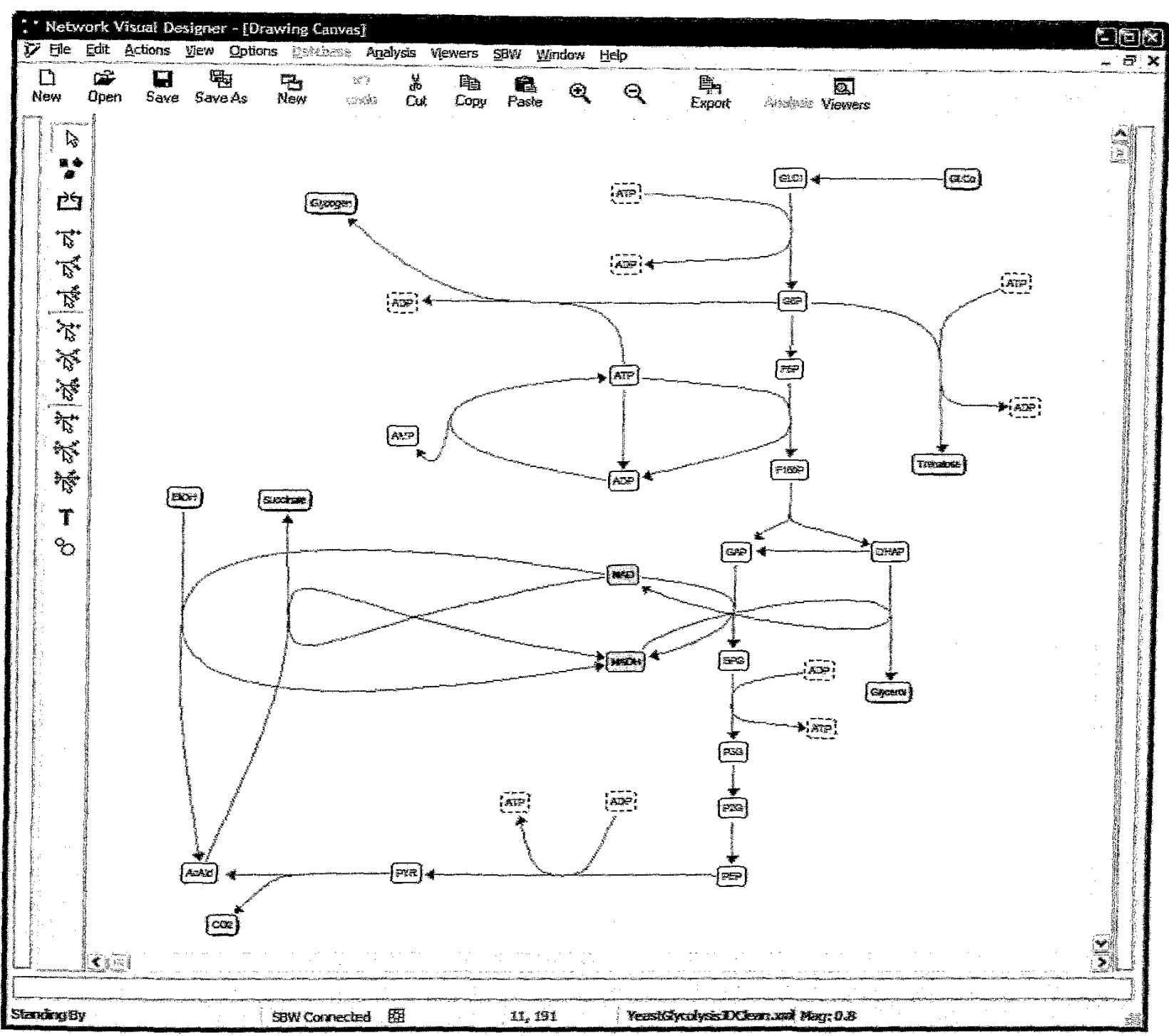

FIG. 10. JDesigner, illustrating a model of glycolysis taken from Pritchard and Kell (2002).

Interaction of JDesigner with Jarnac, or any other compatible simulator, is automatic. Figure 11 illustrates a simulation displayed by IDesigner but actually carried out by Jarnac via SBW.

\section{SBWMetatool}

METATOOL (Pfeiffer et al., 1999) is an application developed by Stefan Shuster, Thomas Pfeiffer, and more recently by Ferdinand Moldenhauer and Juan Carlos Nuno (www.bioinf.mdcberlin. de/projects/metabolic/metatool/). Its primary task is the determination of elementary modes (Schuster et al., 2000), but it also has a variety of other functions, including null space computation and conservation analysis. It easily runs on Linux or Windows, or for that matter any platform that can compile standard C code. METATOOL generates a multitude of information, including, but not exclusively, the null space of the stoichiometry matrix, conservation relations, and what METATOOL was specifically designed to generate, elementary modes. Generating elementary modes is a non-trivial exercise, and other packages, such as the interactive simulator, Jarnac (Sauro, 2000), employ METATOOL for this task.

To make METATOOL available to SBW, we wrote a small controlling application that has an interface to SBW and controls the running of METATOOL. 


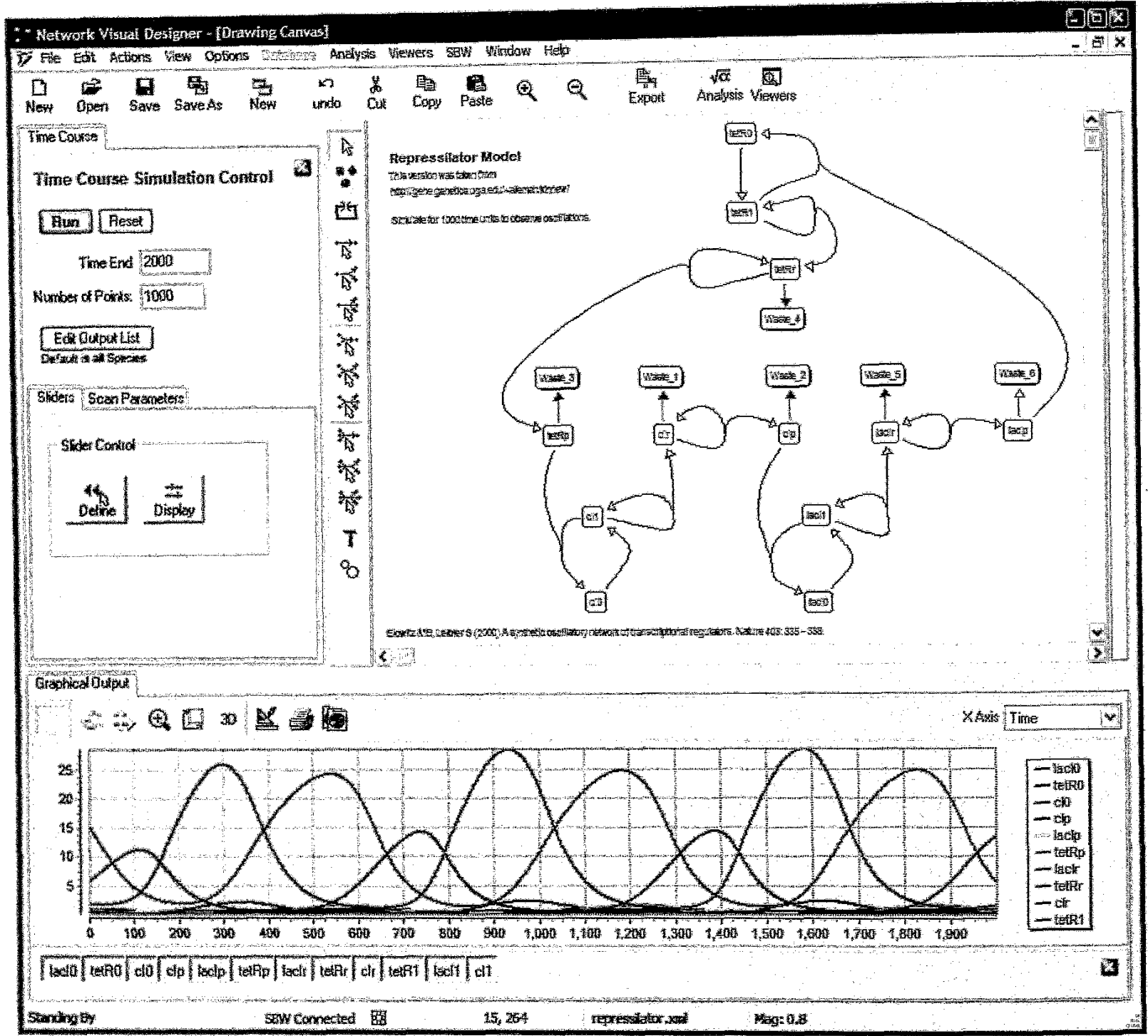

TG. 11. Destgner and Jamac working together to carry out and display the results from a simulation. The model was uken from Elowitz and Leibler (2000), which illustrates a synthetic oseillatory circuit that was constructed in Escherictio colt.

Figure 12 illustrates the interaction of METATOOL with JDesigner. JDesigner acts as the model editor from which users can initiate simulation and METATOOL analysis. The figure illustrates two aspects. The lower panel shows the SBWMetatool interface; this displays all the elementary modes that METATOOL found for the displayed model (Calvin Cycle). Note that one of the elementary nodes in the lower panel is highlighted. The main canvas shows the Calvin reaction network, and the selected elementary mode is displayed on the reaction network by highlighting the appropriate reactions. This allows a user to easily visualize each elementary mode in turn. The example illustrates the ability of SBW to combine wo unrelated applications (JDesigner and METATOOL) and deliver completely new functionality. The ofher point to make is that METATOOL was not modified in this project; we only wrote a small separate SBW-based module that could control the running of METATOOL. 


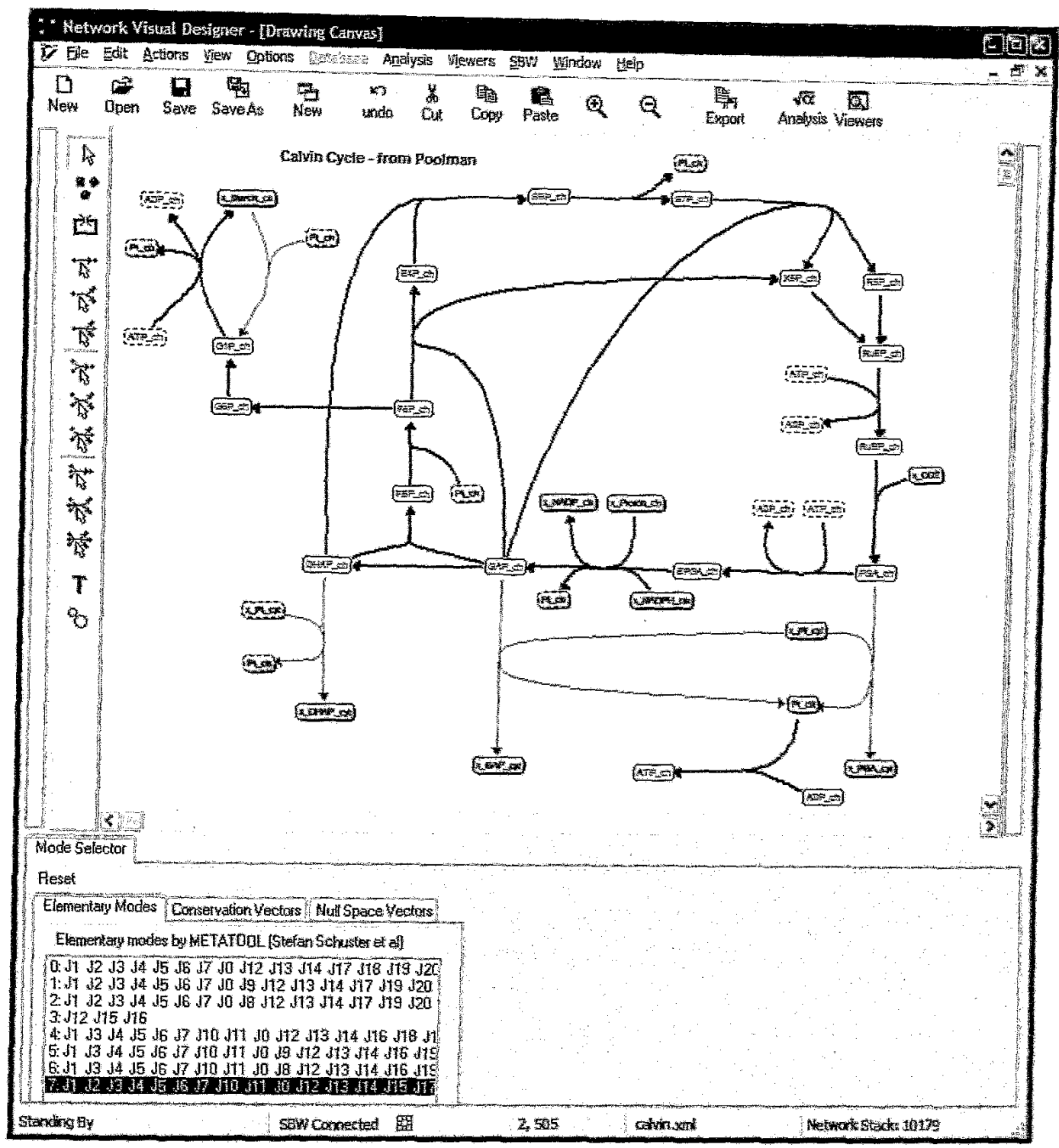

FIG. 12. Operation of METATOOL with JDesigner illustrating the visualization of elementary modes in the Calvit cycle (Poolman et al., 2003). (Model courtesy of M. Poolman, D. Fell, and C. Raines.)

\section{CONCLUSION}

Software reuse is considered a well-known technique for increasing development productivity, but the promise often falls short of the expectation. There are some success stories; in particular, the number of reusable components for Delphi (Borland) and Visual Basic (Microsoit) run into the thousands (www.torry,com) and probably many more for Visual Basic. The question is why have some development environments been more successful than others at encouraging a vibrant community of code reuse? One of the distinguishing features of VB and Delphi development is the ease with which it is possible to develop stand-alone reusable components. Other environments such as CORBA or basic COM have a much steeper leaming curve, and thus the number of people actively engaged in supporting code reuse is correspondingly smaller. For code reuse to be a actively supported, code development should be correspondingly easy to accomplish.

In terms of SBW, we have tried to achieve this situation by making the development of reusable SBW modules as easy as development under VB or Delphi. Developing reusabte modules in Java or Delphi is particulary straightorward under SBW. 


\section{SAURO ET AL.}

In terms of our own development, code reuse was most successful. In developing IDesigner, we did not need to write yet-another-simulator; instead, we leveraged the existing simulator Jarnac. As an example of third-party component use, we were able to wrap METATOOL into a SBW-compliant tool, thus making available the sophisticated algorithms present in METATOOL to all SBW modules.

Some of the key advantages of SBW over other technologies is performance, simplicity of implementation, and language and platform neutrality. With the rapid rise in interest in Systems Biology, it is fair to say that it it probably impossible for one person or even a group to attempt to write the allsinging, all-dancing software tool for Systems Biology, simply because the breadth of the field is too wide.

One great advantage of SBW is that it does not constrain developers to a single platform or even a single language. It eliminates language and platform wars at a stroke, which means we can concentrate on functionality instead.

\section{FUTURE DIRECTIONS}

The future direction of SBW is in two places, enhancements to the core SBW technology, that is enhancements to the Broker and/or binding libraries and enhancements to modules.

\section{Module development}

Module development is taking place on two levels: enhancements to existing modules and development of new modules. The existing modules, in particular JDesigner will continue to be enhanced. One of the most interesting projects is the development of library based model construction. That is, models can be developed in parts and combined at a later date.

As for new modules, two are currently planned for development, this includes an optimization module and a bifurcation module. Both modules are being primarily developed for BioSPICE and will be made available to BioSPICE via the SBW/BioSPICE bridge.

\section{Core development: broker, language bindings, and BioSPICE integration}

The first version of SBW is complete and in production. The current plans for the development of the core are fairly limited. There are a couple of items that we would like to include in a future version. For example, we wonld like to add an additional type to the core data types that can be transmitted from module to module, this type being the complex number type. Since SBW is primarily aimed at the scientific community, complex numbers would prove a very useful addition. One of the primary applications of complex numbers in systems biology is stability analysis and data analysis such as principal component analysis. Of course, in the current version, complex numbers can be transmitted by combining existing types; however, since complex numbers are fundamental to quantitative science there is no reason why they should not have "first class" status as one of the fundamental types.

A second addition we would like to make is to give the binding libraries the ability to decide whether to compress messages before transmission. Some messages especially those containing XML data can be very large. These messages, by their nature are also highly compressible. It would seem sensible therefore to be able to compress such messages automatically before they get transmitted to the receiver. Depending on where the threshold is set to compress a message according to its size, performance increases could easily be achieved.

The most immediate project however to the SBW core is the development of a bridge between SBW and BtoSPICE. This is currently underway and should be completed very soon. As previously mentioned, the bridge will allow modules in both SBW and BioSPICE to communicate with each other. At the moment, SBW and BioSPICE are complimentary in functionality, and such a bridge would therefore greatly benefit both communities. 


\section{NEXT GENERATION SIMULATION TOOLS}

\section{ACKNOWLEDGMENTS}

This work was initially funded by the Japan Science and Technology Corporation under the ERATO Kitano Systems Biology Project. The development of JDesigner and Jarnac were partially funded by ERATO and the Keck Graduate Institute. More recent support for H.M.S. and C.W. was received via a grant awarded from the DARPA/IPTO BioCOMP program, contract number MIPR 03-M296-01. We wish to acknowledge Mark Borisuk, Mineo Morohashi, and Tau-Mu Yi for support, comments, and advice, and the BioSPICE team at SRI and Berkeley for their invaluable assistance in enabling BioSPICE/SBW integration.

\section{REFERENCES}

BIOSPICE. (2001). The BioSPICE Development Project [On-line]. Available: www.biospice.org/.

BOX, D., EHNEBUSKE, D., KAKIVAYAT, G., et al. (2000). Simple object access protocol (SOAP) 1.1: W3C note 08 May 2000 [On-line]. Available: www.w3.org/TR/SOAP/.

BURNS, J.A. (1971). Studies on Complex Enzyme Systems [Ph.D. dissertation]. University of Edinburgh. Available: www.cds.caltech.edu/hsauro/Burns/jimBums.pdf.

CHANCE, B., HIGGINS, J.J., and GARFINKEL, D. (1962). Analogue and digital computer representations of biochemical processes. Proc. Fed. Am. Soc. Exp Biol. 21, 75-86.

ELOWITZ, M.B., and LEIBLER, S. (2000). A synthetic oscillatory network of transcriptional regulators. Nature 403, 335-338.

ENDY, D., and BRENT, R. (2001). Modeling cellular behavior. Nature 409, 391-395.

FELL, D.A., and SAURO, H.M. (1990). Metabolic control analysis by computer: progress and prospects. Biomed. Biochim. Acta 8/9, 811-816.

GARFINKEL, D. (1968). A machine-independent language for the simulation of complex chemical and biochemical systems. Comput. Biomed. Res. 2, 31-44.

HEDLEX, W.J., MELANIE, N.R., BULLIVANT, D.P., et al. (2001). A short introduction to CellML. Phil. Trans. R. Soc. Lond. A 359, 1073-1089.

HUCKA, H.M., FINNEY, A., SAURO, H.M., et al. (2002). The ERATO Systems Biology Workbench: enabling interaction and exchange between software tools for computational biology. Pac. Symp. Biocomput. 450-461.

HUCKA, M., FINNEY, A., SAURO, H.M., et al. (2003). The Systems Biology Markup Language (SBML): a medium for representation and exchange of biochemical network models. Bioinformatics 19, 524-531.

MENDES, P. (1993). Gepasi: a software package for modelling the dynamics, steady states and control of biochemical and other systems. Comput. Appl. Biosei. 9, 563-571.

OLSON, M. and OGBUJ, U. (2002). The Python web services developer messaging technologies compared IOnline]. Available: www-106.jbm.com/developerworks/webservices/library/ws-pyth9/.

OMG. (2001). CORBA specication [On-line]. Available: www.ong.org.

ORAM, A. (2001). Peer-to-Peer: Harnessing the Power of Disruptive Technologies (O'Reilly \& Associates, Sebastopol, CA).

PARK, D.J.M., and WRIGHT, B.E. (1973) Metasim, a general purpose metabolic simulator for studying cellular transformations. Comput. Prog. Biomed. 3, 10-26.

PFEIFFER, T., SANCHEZ-VALDENEBRO, I., NUNO, J.C., et al. (1999) Metatool: for studying metabolic networks. Bioinformatics 15, 251-257.

POOLMAN, M.G., FELL, D.A., and RAINES, C.A. (2003). Elementary modes analysis of photosynthate metabolism in the chloroplast stroma. Eur. J. Biochem. 270, 430-439.

PRITCHARD, L, and KELL, D.K. (2002). Schemes of ftux control in a model of Saccharomyces cerevisiae glycolysis. Eur. J. Biochem. 269, 3894-3904.

RAO, D.M.W., and ARKIN, A.P. (2002). Control ${ }_{x}$ exploitation and tolerance of intracellular noise. Nature 420, $231-237$.

SAURO, H.M. (2000). Jamac: a system for interactive metabolic analysis. In: Animating the Cellular Map: Proceedings of the 9 th International Meeting on BioThermoKinetics. J.H.S. Hoimeyr, JM. Rohwer, and J.L. Snoep, eds. (Stellenbosch University Press), pp. 221-228.

SAURO, H.M., and FELL, D.A. (1991). Scanp: a metabolic simulator and control analysis program. Math. Comput. Modelling 15, 15-28.

SCHUSTER, S., FELL, D.A., and DANDEKAR, T. (2000). A general definition of metabolic pathways useful for systematic organization and analysis of complex metabolic networks. Nat. Biotechnol. 18, 326-332. 


\section{SAURO ET AL.}

TYSON, J.J., CHEN, K.C., and NOVAK, B. (2003). Sniffers, buzzers, toggles and blinkers: dynamics of regulatory and signaling pathways in the cell. Curr. Opin. Cell Biol. 15, 221-231.

WINER, D. (2001). XML-RPC [On-line]. Available: www.xmlrpc.com/spec/.

Address reprint requests to:

Dr. Herbert M. Sauro

Keck Graduate Institute

535 Watson Drive

Claremont, CA 91711

E-mail: hsauro@kgi.edu 